\title{
Discovering the true identity and function of mes- enchymal stem cells
}

\author{
Yo Mabuchi'1,2), Diarmaid D. Houlihan ${ }^{3)}$, Hideyuki Okano1) \\ and Yumi Matsuzaki ${ }^{1, *)}$ \\ 1)Department of Physiology, Keio University School of Medicine, Tokyo, Japan \\ 2)Department of Biochemistry and Biophysics, Graduate School of Health Care Sciences, Tokyo Medical and \\ Dental University, Tokyo, Japan \\ ${ }^{3)}$ Centre for Liver Research, NIHR Biomedical Research Unit, University of Birmingham, Birmingham, UK
}

\begin{abstract}
Mesenchymal stem cells (MSCs) are currently defined as cells that undergo sustained in vitro growth and can give rise to multiple mesenchymal lineages. Traditional MSCs isolation methods require prolonged in vitro culture on plastic plates, which reduces their differentiation potential and proliferative ability. Furthermore, this process alters MSCs-phenotype, making it difficult to identify specific MSCs-markers that could be used for their in vivo localization and prospective isolation. These limitations have hindered investigations into the biology and function of MSCs. This review article focuses on recent developments in the MSC-research field including the identification of novel surface markers for the prospective isolation of both murine and human MSCs. Prospectively isolated MSCs are more proliferative than MSCs prepared by conventional plastic adherence, provide a better substrate for studying MSCs biology and have more potential for regenerative therapy.
\end{abstract}

Rec.6/4/2012, Acc.7/9/2012, pp146-151

\footnotetext{
* Correspondence should be addressed to:

Yumi Matsuzaki, Department of Physiology, Keio University School of Medicine, 35 Shinanomachi, Shinjuku-ku, Tokyo 160-8582, Japan. Phone: +81-3-5363-3117, Fax: +81-3-5363-3566, E-mail: pentadou@gmail.com
}

Key words mesenchymal stem cells, prospective isolation, flow cytometer, physiological function, stem cell niche

\section{Introduction}

The bone marrow (BM) compartment contains both hematopoietic stem cells (HSCs) and non-hematopoietic stem cell populations. HSCs are commonly used as cell therapy for a wide range of diseases. The non-hematopoietic stem cell population, identified as mesenchymal stem cells (MSCs), is believed to support not only hematopoiesis, but also differentiate along various mesodermal lineages to generate fat, bone and cartilage. Because of this multipotency, MSCs are an attractive therapeutic candidate to promote repair or regeneration of damaged tissues of mesenchymal origin ${ }^{1)}$. Additionally their inherent immunosuppressive potential has been utilized to treat a wide variety of autoimmune conditions ${ }^{2)}$. 


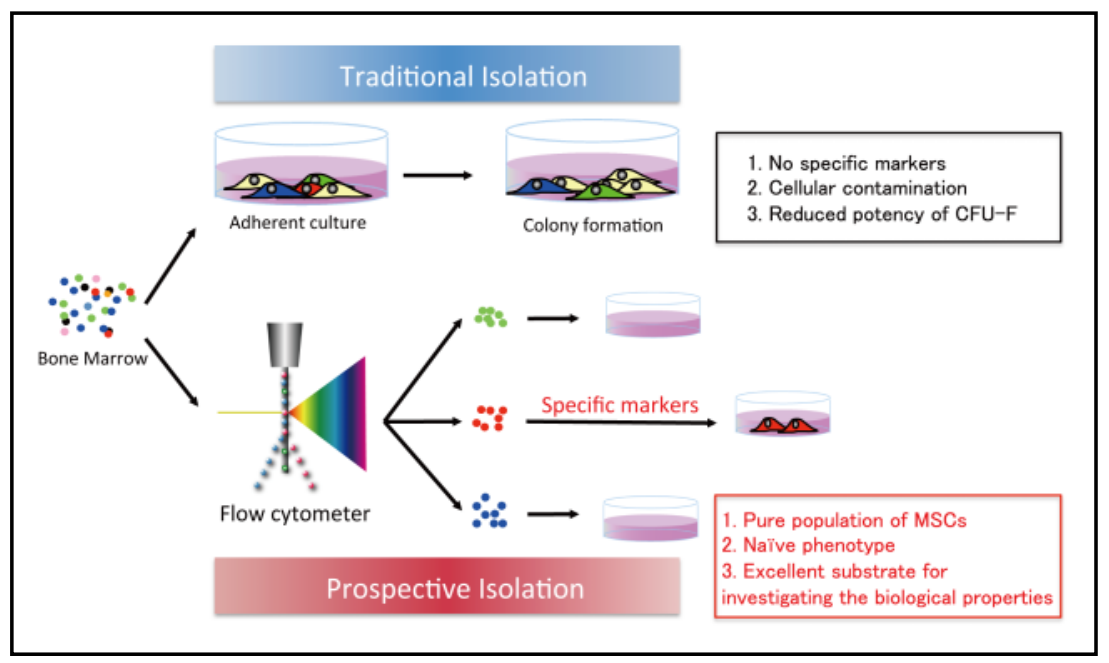

\section{Fig.1 Prospective isolation of Mesenchymal Stem Cells}

Traditional MSC isolation by plastic adherence (Top). Following a period of prolonged culture the majority of the contaminating cells have been washed away or outgrown, enriching for CFU-Fs (Colony formation). The MSCs have a spindle shaped morphology and are capable of differentiating into adipocytes, chondrocytes and osteoblasts in vitro. The prospective isolation of MSCs using specific markers by flow cytometry (Bottom). This method allows the isolation of a pure population of potent MSCs and avoids cellular contamination.
Despite the large number of clinical studies currently investigating the therapeutic potential of MSCs, the method for their isolation has changed little from that used for their initial identification ${ }^{3)}$. Isolating MSCs by plastic adherence has several limitations. Firstly, despite a period of prolonged culture, MSCs populations frequently contain contaminating cells. Secondly, the differentiation potential and proliferative ability of the colony forming unit-fibroblasts (CFUFs) gradually diminishes as the cells acquire a more mature phenotype. Finally, there are also concerns that following prolonged culture, MSCs may acquire chromosomal abnormities pre-disposing them to malignant transformation ${ }^{4)}$. From a scientific perspective very little information exists about the in vivo identity and biological function of MSCs within their niche. In an effort to overcome these problems, there has been an intense effort to identify reliable MSCssurface markers that can facilitate the prospective isolation of colony-initiating cells (Fig.1).

This review summarizes the history of MSCs research, focusing on their identification in different species, including human and mouse. We focus on the recent identification of specific murine and human MSCs-surface markers that have facilitated their in vivo localization and prospective isolation. Finally, we summarize the evidence supporting a physiological role of MSCs in their BM niche.

\section{Historical perspective}

Dr. Fridenstein and his colleagues were the first to document the presence of BM-derived plastic adherent cells that generated CFU-Fs when plated as single cells in vitro ${ }^{5)}$. His group subsequently demonstrated these cells could be readily differentiated into bone, cartilage and fat in vitro. At approximately the same time, Dr. Reddi and colleagues were subcutaneous implanting biological matrix of the shafts of long bones of rats to allogenic recipients ${ }^{6)}$. They found after a period of time that bone and cartilage formed on the implant, and that the resulting bony ossicle subsequently supported hematopoiesis in vivo. These data provided the first evidence that the BM contains stromal progenitors and that these cells may pay a critical role promoting the homing and maintenance of the hematopoietic progenitors and supporting hematopoiesis. Largely based on these studies, Dr. Caplan first used the term 'MSCs' to describe these cells in 19917). Despite intense research into MSCs-biology since that time, very little has been uncovered until recently about their anatomical localization, physiological function or the stromal hierarchy.

\section{What defines the MSC?}

The Tissue Stem Cell Committee of the International Society for Cellular Therapy has proposed minimum criteria that define the human $\mathrm{MSCs}^{8}$. Firstly, the cell must be plastic adherent when cultured under standard conditions. Secondly, MSCs must express CD73, CD90 and CD105, without expression of CD45, CD34, CD14 or CD11b, CD79 or CD19 and HLA-DR surface markers ${ }^{9}$. Finally, MSCs must be capable of differentiating into osteoblasts, adipocytes and chondrocytes in vitro. While this statement clarifies somewhat the cellular characteristics of human MSCs, the situation remains unclear for murine MSCs. Until recently there have been no specific surface markers for murine MSCs. Isolation of murine MSCs therefore has re- 
lied on the isolation of a plastic adherent cell population, with typical morphology, that is capable of tri-lineage differentiation. The definition of both murine and human MSCs has however generated controversy. The classic definition of a stem cell requires that it possess unlimited self-renewal ability and plasticity. To this end serial transplantation experiments demonstrating that infused stem cells give rise to terminally differentiated daughter tissue cells while maintaining their naive phenotype could provide proof of concept. MSCs have until recently fallen far short of meeting these criteria as the execution of such assays requires specific cell markers that can be used for in situ localization and prospective MSC isolation. The traditional MSC isolation technique by plastic adherence alters the MSCs' phenotype preventing the identification of such specific surface markers ${ }^{10)}$.

\section{Identification of murine MSC markers}

The identification of specific MSC markers began with the observation that hematopoietic and mesenchymal lineage cells are derived from individual lineage-specific stem cells ${ }^{11)}$. Furthermore, a series of developmental studies demonstrated that a proportion of adult BM MSCs may have a developmental origin in the neural crest ${ }^{12,13)}$. Based on these observations we carried out a detailed screening of numerous candidate molecules and PDGFR $\alpha$ and Sca-1 were identified as specific markers of murine MSCs ${ }^{14)}$. PDGFR $\alpha^{+} /$Sca- $1^{+}(\mathrm{P} \alpha \mathrm{S})$ cells fulfill the basic requirements of the definition of MSCs, in that they are capable of unlimited self-renewal and differentiate into osteoblasts, chondrocytes and adipocytes under appropriate conditions in vitro. $\mathrm{P} \alpha \mathrm{S}$ cells proliferate almost without senescence when cultured on plastic, yielding more than $10^{7}$ cells from the original 5000 cells seeded with a doubling time of 50.6 hours. When compared to traditional isolation methods (plastic adherence) the $\mathrm{P} \alpha \mathrm{S}$ cells showed a 120,000-fold higher CFU-F frequency than unfractionated bone marrow mononuclear cells. Moreover, $\mathrm{P} \alpha \mathrm{S}$ cells reside in the perivascular space adjacent to vascular smooth muscle and express Angiopoietin-1 and CXCL12 suggesting that MSCs constitute part of the hematopoietic niche. Finally in an elegant demonstration of the stemness, $1 \times 10^{4}$ freshly isolated $\mathrm{P} \alpha \mathrm{S}$ cells were intravenously injected into lethally irradiated recipient mice. These cells homed to their niche in the BM expressing niche factors (Angiopoietin-1 and CXCL12) and also differentiated into osteoblasts and adipocytes in vivo. Sixteen weeks following the transplantation the mice were sacrificed and the isolated $\mathrm{P} \alpha \mathrm{S}$ cells were capable of forming CFU-Fs and tri-lineage differentiation. Thus, this paper was the first to identify specific murine MSC surface markers and convincingly show the in vivo self-renewal and multipotency of $\mathrm{P} \alpha \mathrm{S}$ MSCs.

More recently the neural marker Nestin has been used to identify and prospectively isolate murine MSCs ${ }^{15}$. Nestin ${ }^{+}$ cells represent a small subset of stromal cells in BM. These cells are anatomically located in the perivascular space, adjacent to catecholaminergic nerve fibers and HSCs. Similar to $\mathrm{P} \alpha \mathrm{S}$ MSCs they express niche factors (ADRB3 and CXCL12 expression) and are capable of differentiating into osteoblasts, chondrocytes and adipocytes in vitro and in vivo. Nestin ${ }^{+}$MSCs also play an important role in maintaining the HSC niche. Depletion of Nestin+ MSCs led to a dramatic reduction in the number and function of HSCs. Additionally knockout mice had significantly impaired homing of transplanted HSCs back to their BM niche. These data support an important role of MSCs in the maintenance of the HSC niche.

These are the first studies to identify specific markers that can be used for prospective isolation and in vivo localization of MSCs. In addition $\mathrm{P} \alpha \mathrm{S}$ and Nestin ${ }^{+}$MSCs have been assayed in the traditional stem cell assays (including serial transplantation assays and clonogenic assays) and their properties of self-renewal and potency are now confirmed. We have also gained a valuable insight into the importance of these cells in maintaining the HSCs niche. Without doubt $\mathrm{P} \alpha \mathrm{S}$ and Nestin+ cells will provide an invaluable substrate for ongoing research into biological function, hierarchical structure and therapeutic potential of MSCs. It is not entirely clear if Nestin+ cells are the same as $\mathrm{P} \alpha \mathrm{S}$ cells. We know that $\mathrm{Nestin}^{+}$cells largely overlap the PDGFR $\alpha+{ }^{+} \mathrm{CD} 51^{+}$population. This population contains Sca-1 positive and negative cells (personal communication: Frenette PS). These data suggest that the Nestin ${ }^{+}$population is comprised of $\mathrm{P} \alpha \mathrm{S}$ and $\mathrm{PDGFR} \alpha^{+}$cells. Interestingly, investigations using nestin-Cherry and nestin-GFP double transgenic mice demonstrates nestin-Cherry expression around larger vessels but not around sinusoids, while nestin-GFP expression was detected around both ${ }^{16}$. Thus, different nestin transgenes seem to be expressed by different subpopulations of perivascular stromal cells. 


\section{Prospective isolation of human MSCs}

Numerous surface markers of human MSCs including CD49a, CD56, CD63, CD73, CD105, CD106, CD140b, CD271, MSCA-1, Stro-1 and SSEA4 have been identified $^{9}$. Many of these markers have been used singly or in combination to enrich for CFU-F in human BM and avoid cellular contamination. Unfortunately, many of these markers are widely expressed in stromal cells and lack specificity, contributing to the significant heterogeneity seen among clonogenic colonies from single isolations. Moreover many of these markers do not help uncover the true identity and function of MSCs in vivo. Currently there is a need to identify specific human MSC markers to identify MSCs in situ, perform assays of self-renewal and multipotency, and to begin to probe their biological function.

It is clear that prolonged culture on plastic reduces the differentiation potential and proliferative ability of the CFUFs as the cells acquire a more mature phenotype ${ }^{17)}$. This is accompanied by cell senescence and potentially reduced therapeutic potential. A variety of different methods have been used to over come this issue including supplementing culture media with growth factors, culture in hypoxic conditions and culture in non-adherent conditions. For example, Prockop and colleagues demonstrate that human MSCs cultured as 3D spheroids have enhanced anti-inflammatory properties in a model of peritonitis. The cultured cells were also smaller, allowing them to escape from the lung circulation and migrate to a variety of different organs ${ }^{18)}$. Additionally, the longterm culture of MSCs in hypoxic conditions maintains these cells in an undifferentiated and multipotent state ${ }^{19}$. There is little doubt that the culture conditions used to expand MSCs prior to their clinical use have a dramatic effect on their therapeutic potential.

CD146 has provided valuable insight into the in vivo localization and function of human $\mathrm{MSCs}^{20}$. It marks an adventitial reticular cell that resides in the endothelial space in human BM. These cells express typical stromal markers (CD105, CD49a, CD63, CD90, CD140b) and are capable of robust tri-lineage differentiation. Additionally, subcutaneous transplantation of $\mathrm{CD} 146^{+}$clonogenic cells into immunodeficient mice resulted in formation of boney ossicles, sinusoidal vasculature and finally functioning hematopoiesis. Furthermore, a small proportion of the infused cells localized to the murine HSC niche and expressed supporting factors including Ang-1. As a clear demonstration of the self-renewal potency of these cells, the transplanted CD146+ MSCs were re-isolated, cultured and formed CFUFs capable of tri-lineage differentiation.

Traditionally, MSCs were thought to reside only within the BM as the stromal counterpart to HSCs. Interestingly, the utility of CD146 as a prospective marker of human MSCs is not limited to adult human BM. Crisan et al. used immunohistochemistry to examine various different tissue types including both adult and fetal human skeletal muscle, pancreas, adipose tissue, and placenta and identified CD146, NG2 and PDGF $\alpha$ as prospective pericyte markers ${ }^{21}$. They observed some striking similarities between pericytes and BM MSCs. Flow cytometry was used to derive a pure population of pericytes from each tissue type. These cells were then differentiated into muscle, bone, fat and cartilage using the standard assays used for MSCs. Furthermore, following long-term culture, pericytes expressed typical MSCs markers including CD73, CD90 and CD105 and migrated in a culture model of chemotaxis. These data clearly identify CD146 as a specific marker of mesenchymal progenitor cells in a wide range of organs.

\section{Role of murine MSCs in the bone marrow niche}

The HSC niche provides a specialized microenvironment that promotes stem cell maintenance and function. Several cells types including osteoblasts, endothelial cells, and adventitial reticular cells have been proposed to secrete a wide variety of signals that contribute to the niche function ${ }^{22)}$. It has been speculated that MSCs may play an important function in this niche, however until recently their contribution has remained unproven. The observation by Morikawa et al. that $\mathrm{P} \alpha \mathrm{S}$ cells reside in the perivascular space adjacent to HSCs, and express Angiopoietin-1 and CXCL12 supports this hypothesis (Fig.2). Indeed, Nestin+ MSCs appear to play a critical role not only the maintenance of HSCs within the niche, but also the homing of transplanted HSCs back to the BM. Interestingly, a significant number of perivascular PDGFR $\alpha$-positive cells express Nestin and the exact contribution of each subpopulation is not entirely clear. Recent data suggests that other cell types including non-myelinating Schwann cells play an important role in maintaining the HSC niche via modulation of TGF- $\beta^{23)}$. Additionally Morrison et al. confirmed the importance of stem cell factor (Scf) in maintaining the HSC niche and demonstrated that Scf was primarily ex- 


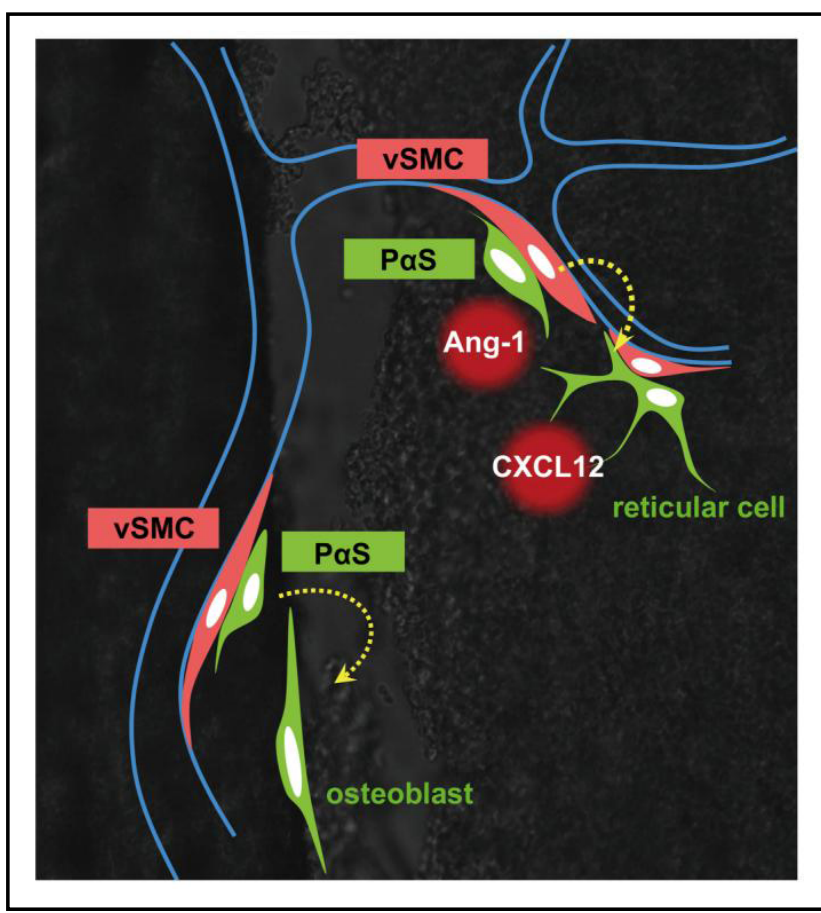

Fig.2 Schematic model of the physiological localisation and behavior of MSCs in BM

$\mathrm{P} \alpha \mathrm{S}$ cells located in the arterial perivascular space in association with vascular smooth muscle cells (vSMCs) give rise to osteoblasts and reticular cells that function as vascular niche cells, by producing major chemo-attractants (CXCL12 and Ang-1) for hematopoietic stem cells.

pressed by perivascular cells throughout the $\mathrm{BM}^{16)}$. HSC frequency and function were not affected when Scf was conditionally deleted from hematopoietic cells, osteoblasts, Nestin ${ }^{+}$cells. However, HSCs were depleted from BM when Scf was deleted from endothelial cells or leptin receptor (Lepr)-expressing perivascular stromal cells (also positive for PDGFR $\alpha, \operatorname{PDGFR} \beta, \mathrm{CXCL} 12$ and alkaline phosphatase expression). Clearly much remains unknown in this complex microenvironment, however the data suggests that one or more MSCs subtypes contribute to HSCs niche homeostasis.

\section{Concluding remarks}

The hypothesis that a rare population of multipotent stromal stem cells or MSCs, capable of generating all stromal subtypes, existed in BM was greeted with almost universally approval in the scientific world. Proposed physiological functions of MSCs included maintenance of the HSCs niche, replenishment of mesenchymal tissue, and wound healing and repair. Interrogating the biology and function of MSCs was significantly delayed however by a lack of specific cell markers. Recently however, the field has taken a significant leap forward, with the identification of $\mathrm{P} \alpha \mathrm{S}$ and Nestin+ MSCs. These markers have not only allowed us to confirm the stem cell properties of these cells, but also interrogate their biological functions including maintenance of the HSCs niche. The prospective isolation of MSCs using selective markers is crucial if we are to address the many unanswered scientific questions related to these cells.

\section{Source of funding}

The Project for Realization of Regenerative Medicine (to Y. Matsuzaki and H.O.), a Grant-in-Aid for Encouragement of Young Medical Scientists from Keio University (to Y. Mabuchi), a Grant-inAid for Scientific Research (KAKENHI) (to Y. Mabuchi), and a grantin-aid from the Global Century COE program of the MEXT, Japan to Keio University. D.H. is funded by the Medical Research Council, UK.

\section{Disclosure of conflict of interests}

The authors declare no competing financial interests.

\section{References}

1) Stappenbeck TS, Miyoshi $\mathrm{H}$ : The role of stromal stem cells in tissue regeneration and wound repair. Science. 2009; 324: 1666-1669.

2) Liu Y, Wang L, Kikuiri T, Akiyama K, Chen $C, X u X$, Yang R, Chen W, Wang S, Shi S: Mesenchymal stem cell-based tissue regeneration is governed by recipient T lymphocytes via IFN- $\gamma$ and TNF- $\alpha$. Nat Med. 2011; 17: 1594-1601.

3) Pittenger MF, Mackay AM, Beck SC, Jaiswal RK, Douglas R, Mosca JD, Moorman MA, Simonetti DW, Craig S, Marshak DR: Multilineage potential of adult human mesenchymal stem cells. Science. 1999; 284: 143-147.

4) Ben-David U, Mayshar Y, Benvenisty N: Large-scale analysis reveals acquisition of lineage-specific chromosomal aberrations in human adult stem cells. Cell Stem Cell. 2011; 9: 97-102.

5) Friedenstein AJ, Deriglasova UF, Kulagina NN, Panasuk AF, Rudakowa SF, Luriá EA, Ruadkow IA: Precursors for fibroblasts in different populations of hematopoietic cells as detected by the in vitro colony assay method. Exp Hematol. 1974; 2: 83-92.

6) Reddi $\mathrm{AH}$, Huggins $\mathrm{CB}$ : Formation of bone marrow in fibroblast-transformation ossicles. Proc Natl Acad Sci 
USA. 1975; 72: 2212-2216.

7) Caplan Al: Mesenchymal Stem-Cells. Journal of Orthopaedic Research. 1991; 9: 641-650.

8) Dominici M, Le Blanc K, Mueller I, Slaper-Cortenbach I, Marini FC, Krause DS, Deans RJ, Keating A, Prockop DJ, Horwitz EM: Minimal criteria for defining multipotent mesenchymal stromal cells. The International Society for Cellular Therapy position statement. Cytotherapy. 2006; 8: 315-317.

9) Mabuchi Y, Morikawa S, Suzuki S, Sunabori T, Okano $\mathrm{H}$, Matsuzaki Y: Prospective isolation and identification of human mesenchymal stem cell by flow cytometry. Inflamm Regen. 2009; 29: 73-78.

10)Bianco P, Robey PG, Simmons PJ: Mesenchymal stem cells: revisiting history, concepts, and assays. Cell Stem Cell. 2008; 2: 313-319.

11) Koide $Y$, Morikawa $S$, Mabuchi $Y$, Muguruma $Y$, Hiratsu E, Hasegawa K, Kobayashi M, Ando K, Kinjo K, Okano $\mathrm{H}$, Matsuzaki $\mathrm{Y}$ : Two distinct stem cell lineages in murine bone marrow. Stem Cells. 2007; 25: 1213-1221.

12)Morikawa S, Mabuchi $Y$, Niibe K, Suzuki S, Nagoshi $\mathrm{N}$, Sunabori T, Shimmura S, Nagai Y, Nakagawa T, Okano H, Matsuzaki Y: Development of mesenchymal stem cells partially originate from the neural crest. Biochem Biophys Res Commun. 2009; 379: 1114-1119.

13) Takashima $Y$, Era T, Nakao K, Kondo S, Kasuga M, Smith AG, Nishikawa S: Neuroepithelial cells supply an initial transient wave of MSC differentiation. Cell. 2007; 129: 1377-1388.

14) Morikawa S, Mabuchi $Y$, Kubota $Y$, Nagai $Y$, Niibe K, Hiratsu E, Suzuki S, Miyauchi-Hara C, Nagoshi N, Sunabori T, Shimmura S, Miyawaki A, Nakagawa T, Suda T, Okano H, Matsuzaki Y: Prospective identification, isolation, and systemic transplantation of multipotent mesenchymal stem cells in murine bone marrow. J Exp Med. 2009; 206: 2483-2496.

15)Méndez-Ferrer S, Michurina TV, Ferraro F, Mazloom AR, Macarthur BD, Lira SA, Scadden DT, Ma'ayan A, Enikolopov GN, Frenette PS: Mesenchymal and haematopoietic stem cells form a unique bone mar- row niche. Nature. 2010; 466: 829-834.

16)Ding L, Saunders TL, Enikolopov G, Morrison SJ: Endothelial and perivascular cells maintain haematopoietic stem cells. Nature. 2012; 481: 457-462.

17) Kim J, Kang JW, Park JH, Choi Y, Choi KS, Park KD, Baek DH, Seong SK, Min HK, Kim HS: Biological characterization of long-term cultured human mesenchymal stem cells. Arch Pharm Res. 2009; 32: 117-126.

18)Bartosh TJ, Ylöstalo JH, Mohammadipoor A, Bazhanov $\mathrm{N}$, Coble K, Claypool K, Lee RH, Choi H, Prockop DJ: Aggregation of human mesenchymal stromal cells (MSCs) into 3D spheroids enhances their antiinflammatory properties. Proc Natl Acad Sci USA. 2010; 107: 13724-13729.

19) Basciano L, Nemos C, Foliguet B, de Isla N, de Carvalho M, Tran N, Dalloul A: Long term culture of mesenchymal stem cells in hypoxia promotes a genetic program maintaining their undifferentiated and multipotent status. BMC Cell Biol. 2011; 12: 12.

20)Sacchetti B, Funari A, Michienzi S, Di Cesare S, Piersanti S, Saggio I, Tagliafico E, Ferrari S, Robey PG, Riminucci M, Bianco P: Self-renewing osteoprogenitors in bone marrow sinusoids can organize a hematopoietic microenvironment. Cell. 2007; 131: 324336.

21)Crisan M, Yap S, Casteilla L, Chen CW, Corselli M, Park TS, Andriolo G, Sun B, Zheng B, Zhang L, Norotte C, Teng PN, Traas J, Schugar R, Deasy BM, Badylak S, Buhring HJ, Giacobino JP, Lazzari L, Huard J, Péault $B$ : A perivascular origin for mesenchymal stem cells in multiple human organs. Cell Stem Cell. 2008; 3: 301313.

22)Wilson A, Trumpp A: Bone-marrow haematopoieticstem-cell niches. Nature Reviews Immunology. 2006; 6: 93-106.

23)Yamazaki S, Ema H, Karlsson G, Yamaguchi T, Miyoshi H, Shioda S, Taketo MM, Karlsson S, Iwama A, Nakauchi H: Nonmyelinating Schwann cells maintain hematopoietic stem cell hibernation in the bone marrow niche. Cell. 2011; 147: 1146-1158. 